\title{
Role of Ovarian Fertility Sparing Surgery (FSS) in Cases of Early Stage 1 Ovarian Cancer in Patients in Reproductive Age Group
}

\author{
Ahmed Essmat \\ Department of Obstetrics and Gynecology, Faculty of Medicine, Alexandria University, Alexandria, Egypt \\ Email: princeessmat@gmail.com
}

How to cite this paper: Essmat, A. (2021) Role of Ovarian Fertility Sparing Surgery (FSS) in Cases of Early Stage 1 Ovarian Cancer in Patients in Reproductive Age Group. Open Journal of Obstetrics and Gynecolo$g y, 11,732-741$.

https://doi.org/10.4236/ojog.2021.116068

Received: May 2, 2021

Accepted: June 15, 2021

Published: June 18, 2021

Copyright $\odot 2021$ by author(s) and Scientific Research Publishing Inc. This work is licensed under the Creative Commons Attribution International License (CC BY 4.0).

http://creativecommons.org/licenses/by/4.0/

\begin{abstract}
Objective: To assess the role of FSS in women with early stage 1 ovarian cancer. Methods: This was a retrospective analytic study of the results of treatment of 24 patients all under 40 years of age who underwent a full conservative staging laparotomy procedure in oncology center of El Shatby Maternity hospital, Alexandria University in the period of one year from October 2019 to September 2020. All patients were followed up for a six month period following surgery by the pre-operatively elevated tumor marker and by a CT abdomen and pelvis to detect any tumor recurrence. Results: The mean age at diagnosis was 24.29 Years. 6/24 (25\%) of surface epithelial tumor were G1, 8/24 (33.3\%) were G2, 4/24 (16.7\%) were G3. 20/24 (83.3\%) of patients were stage FIGO 1a, $4 / 25$ (16.7\%) were stage $1 \mathrm{~b}$, and none of them was stage $1 \mathrm{c}$. $20 / 24(83.3 \%)$ of patients were stage FIGO 1a, 4/25 (16.7\%) were stage $1 \mathrm{~b}$, and none of them was stage 1c. Recurrence was reported in 3/24 of cases (12.5\%), such 3 cases underwent unilateral SO plus a FCSLP. No recurrence was reported in cases of bilateral tumors that underwent unilateral SO and a contralateral cystectomy. $100 \%$ of recurrence was in epithelial tumors. $1 / 24$ (4.1\%) was clear cell, $1 / 24(4.1 \%)$ was serous and $1 / 24$ (4.1\%) was mucinous. None of the endometroid tumors did recur. Also none of the non-epithelial tumors showed any recurrence. Tumors of G1 showed no recurrence, G2 tumors showed 33.3\% recurrence and G3 tumors showed the highest recurrence rate (66.6\%). Conclusion: ovarian FSS is a safe surgical option for nearly all OC patients with low risk of recurrence, with apparently early stage OC, after being confirmed by a FCSLP to exclude any occult metastasis (occult advanced stage OC), including those cases of early epithelial OC, germ cell and gonadal stromal tumors and it should be considered for patients who have a strong desire to keep their fertility.
\end{abstract}




\section{Keywords}

Early Ovarian Cancer, Fertility Sparing Surgery, Fertility Preservation

\section{Introduction}

Ovarian cancer $(\mathrm{OC})$ is a disease of postmenopausal females. Its median age of diagnosis is 63 years. $10 \%$ of cases of ovarian cancer occur in young age [1]. Those patients usually have positive family history of OC, such as cases have positive BRCA1 and BRCA2 gene mutations, that are more prone to develop OC at earlier age usually at their thirties. Yet, these ladies wouldn't complete their family size and they want to preserve their fertility and reproductive potential. Screening of these patients with such gene mutation may allow early detection and hence the early possible intervention [2]. Majority of ovarian cancer is epithelial in origin, which represents about $90 \%$ of all case of ovarian malignancies. Only $5 \%$ to $10 \%$ of OC cases are non-epithelial in origin (sex cord or germ cell types). About $15 \%$ of those patients are candidates for ovarian fertility sparing surgery (FSS) [3].

Malignant ovarian germ cell tumors comprise nearly $5 \%$ of all ovarian malignancies. They typically occur in adolescents and young women. Nearly $60 \%$ are stage I (confined to the ovary). Thus, the surgical management of these tumors can usually consist of unilateral salpingo-oophorectomy ( $\mathrm{SO}$ ) plus surgical staging. In the case of bilateral ovarian dysgerminomas, standard management is bilateral SO but bilateral ovarian cystectomies or unilateral SO plus ovarian cystectomy has been performed in an attempt to preserve a portion of a normal ovary. The current standard practice following surgery is postoperative chemotherapy consisting of the combination of bleomycin, etoposide, and cisplatin (BEP) for all germ cell tumors with exception of dysgerminoma. Chemotherapy carries $30 \%$ risk of ovarian failure and premature menopause. These effects are usually reversible as many patients regain their fertility and successful pregnancies have been reported after primary surgery plus combination chemotherapy [4].

Sex cord-stromal tumors usually occur in postmenopausal females, but juvenile granulosa cell tumor variety usually occurs in younger adolescent girls. It has excellent prognosis as $95 \%$ of cases are discovered in stage 1 . Thus, fertility-sparing surgery is possible in a high percentage of young patients. For patients with primary advanced disease or recurrent disease, platinum-based chemotherapy is the most common treatment [5].

Fertility sparing surgery (FSS) is different surgical procedures that can be performed in younger patients with early cancer stage to preserve and keep their reproductive potential. There are different types of ovarian FSS. These can include ovarian cystectomy, unilateral SO with uterine preservation and bilateral SO with preservation of the uterus which may be later followed by ART to achieve 
pregnancy. These procedures are associated with a full staging laparotomy procedures (FSLPs) which include peritoneal cytology, multiple peritoneal biopsies, infracolic omentectomy and pelvic with or without para aortic lymphadenectomy. [6] Such procedures are important to detect presence of an occult stage 3 cancer i.e. presence of microscopically positive omental, peritoneal, pelvic or para aortic lymph nodes. This finding can be detected in 15 up to $30 \%$ of cases of patients who were originally diagnosed and planned for FSS and after such diagnosis, a further stepping up for management should be planned [7].

The most important prognostic factor of $\mathrm{OC}$ is the tumor stage where the disease is limited to one or both ovaries. Disease diagnosis at younger age have more favorable prognosis. Tumor grade and histology also have an impact on prognosis. Tumors with low grades have more favorable prognosis [8].

\section{Aim of the Work}

To assess the role of FSS in women with early stage 1 ovarian cancer.

\section{Patients and Methods}

\section{Inclusion criteria}

1) Patient aged 40 years or younger.

2) Patients who have a strong desire to preserve their fertility.

3) Cases with clinically (on clinical examination and Ultrasound) and radiologically (in CT scan) stage 1 ovarian cancer.

4) Presence of one or more $M$ features on IOTA scoring system [9].

5) Absence of gross metastatic disease in omentum, peritoneum, pelvic and para aortic lymph nodes.

6) Patients who agree to participate in the study.

Exclusion criteria

1) Presence of gross or macroscopic disease or metastasis in peritoneal surfaces, omentum or lymph nodes.

2) Cases who are clinically or radiologically (in CTscan) proven to be beyond stage 1 OC. e.g. omental cakes or peritoneal metastasis.

3) Absence of patient's desire of future fertility.

4) Pathologically proven micro-metastasis (occult stage $3 \mathrm{OC}$ ), as proved by histopathological analysis of the specimens postoperative i.e. microscopic metastatic implants in previously mentioned sites.

5) Patients who refuse to share in this study.

\section{Methods}

This was a retrospective analytic study (retrospective review) of the results of treatment of 24 patients all under 40 years of age who underwent a full conservative staging laparotomy procedure in oncology center of El Shatby Maternity hospital, Alexandria University in the period of one year from October 2019 to September 2020.

All patients had detailed history taking including demographic data analysis, 
gravity and parity, family history of breast or ovarian cancer. Patients underwent full clinical examination, then detailed ultrasound examination of the abdomen and pelvis to assess the ovarian neoplasm.

IOTA system was used to assess the possibility of malignancy in the ovarian mass. At least one or more $\mathrm{M}$ criteria should be present to include the case in the study. A full tumor marker analysis was done including Ca125, Ca19.9, Ca15.3, CEA, LDH, b hcg and a-FP. Each case had undergone CT abdomen and pelvis to assess omentum, peritoneal surfaces, pelvic and para-aortic lymph nodes as well as to confirm the origin of the ovarian neoplasm

Full conservative staging laparotomy procedure (FCSLP) and FSS:

All cases underwent a lower midline laparotomy, peritoneal cytology, inspection and palpation of all peritoneal surfaces, ascitic fluid aspiration (if ascites was present), random peritoneal biopsies and at least ipsilateral pelvic lymphadenectomy if the mass was unilateral. A further FSS was done and was individualized for each case according to bilaterality of the mass. Unilateral SO was the standard FSS used in our practice. All specimens were sent for histopathological analysis. All patients were followed up for a six month period following surgery by the pre-operatively elevated tumor marker and by a CT abdomen and pelvis to detect any tumor recurrence

\section{Results}

\section{1) Patient characteristics and tumor laterality: (Table 1)}

a) The mean age at diagnosis was 24.29 Years. The patient's age ranged from 14 to 40 years. $50 \%$ of patients were between 19 and 25 years.

b) $54.2 \%$ of our patients were married and $45.8 \%$ were unmarried. $83.4 \%$ of married patients were nullipara and $8.3 \%$ of them had history of previous one or more delivery.

c) $83.3 \%$ of tumors were unilateral, $58.3 \%$ of unilateral tumors were left sided, and $25 \%$ were right sided. Tumors were bilateral in $16.7 \%$ of cases.

2) Tumor histo-pathological type, grade and FIGO stage: (Table 2)

a) Tumor histo-pathology:

$70.7 \%$ of tumor pathology was epithelial in origin. $6 / 24$ (25\%) of epithelial tumors were endometroid, 4/24 (16.6\%) were mucinous, $2 / 24$ (8.3\%) were clear cell type and 1/24 (4.1\%) were of serous in origin. Sex cord stromal tumors were 6/24 (25\%) all were granulosa cell tumors. Germ cell tumors were the least frequent type ... 4/24 (16.7\%), all were immature teratomas.

b) Tumor grading:

6/24 (25\%) of surface epithelial tumor were G1, 8/24 (33.3\%) were G2, 4/24 (16.7\%) were G3 (poorly differentiated type) the remaining tumors had no grading system.

c) Tumor FIGO substage:

20/24(83.3\%) of patients were stage FIGO 1a, 4/25 (16.7\%) were stage $1 \mathrm{~b}$, and none of them was stage $1 \mathrm{c}$. 
Table 1. Patient characteristics and tumor laterality.

\begin{tabular}{|c|c|c|}
\hline & Frequency & Percent \\
\hline \multicolumn{3}{|l|}{ Age } \\
\hline $14-18$ & 6 & 25.0 \\
\hline $19-25$ & 12 & 50.0 \\
\hline $26-40$ & 6 & 25.0 \\
\hline Range & \multicolumn{2}{|c|}{$14.0-41.0$} \\
\hline Mean \pm S.D. & \multicolumn{2}{|c|}{$24.29 \pm 7.97$} \\
\hline \multicolumn{3}{|l|}{ Marital status } \\
\hline married & 13 & 54.2 \\
\hline virgin & 11 & 45.8 \\
\hline \multicolumn{3}{|l|}{ Gravidity } \\
\hline 0 & 20 & 83.4 \\
\hline 1 & 2 & 8.3 \\
\hline 3 & 2 & 8.3 \\
\hline \multicolumn{3}{|l|}{ Parity } \\
\hline 0 & 20 & 83.4 \\
\hline 1 & 2 & 8.3 \\
\hline 3 & 2 & 8.3 \\
\hline \multicolumn{3}{|l|}{ Bilaterality } \\
\hline bilateral & 4 & 16.7 \\
\hline unilateral & 20 & 83.3 \\
\hline \multicolumn{3}{|l|}{ Side } \\
\hline Bilateral & 4 & 16.7 \\
\hline Lt. & 14 & 58.3 \\
\hline Rt & 6 & 25.0 \\
\hline
\end{tabular}

Table 2. Tumor histo-pathological type, grade and FIGO substage.

\begin{tabular}{ccc}
\hline & Frequency & Percent \\
\hline Tumor histopathological type & 13 & 58 \\
Epithelial & 6 & 25 \\
- Endometroid & 4 & 16.6 \\
- mucimous & 1 & 8.3 \\
• Clear cell & 1 & 4.1 \\
- Papillary & 6 & 25.0 \\
Six cord stromal tumor & 4 & 16.7 \\
Germ cell tumour & & \\
Tumor grade & 6 & 25.0 \\
Grade 1 & 8 & 33.3 \\
Grade 2 & 4 & 16.7 \\
Grade 3 & 6 & 25.0 \\
No & & \\
Tumor FIGO substage & 20 & 83.3 \\
1a & 4 & 16.7 \\
1b & 0 & 0 \\
1c & 0 & 0
\end{tabular}


d) Omentum and pelvic lymph node (LN) status:

None of our patients had positive LN, or omental involvement, as proved by histopathological analysis post-operatively.

3) Tumor recurrence: (Table 3 )

$21 / 24(87.5 \%)$ of our patients did not have pelvic or distant recurrence as proved by CT scan and the preoperatively elevated tumor marker levels (all patients had clean CT abdomen and pelvic and normalized preoperatively elevated tumor marker level. Only $3 / 24$ (12.5\%) had recurrence that was detected by CT scan and the preoperatively elevated tumor marker levels.

4) Surgical procedures and assessment of treatment response: (Table 4)

a) Surgical procedures (FSS and FCSLPs): Unilateral SO was the primary surgical treatment that was used in conjunction with the FCSLPs, it was done in 20/24 (84.3\%) of our cases where tumor was unilateral. $4 / 24$ (16.6\%) of patients had Unilateral SO and contralateral cystectomy when cases were masses were bilateral. Again this was associated with a FCSLP as mentioned above.

b) All patients underwent a CT abdomen and pelvic and preoperatively elevated tumor marker level. 21/24 (87.5\%) of patients did not show any recurrence, while $3 / 24$ (12.5\%) had evidence of disease recurrence.

5) Tumor recurrence and its relation to demographic data, tumor laterality, IOTA scoring, type of surgical procedure, tumor HP and tumor grade: (Tables 5-7)

a) Recurrence and demographic data: (Table 5)

The median age of recurrence was 23.3 years. $100 \%$ of tumor recurrence occurred in virgin (unmarried) patient. None of the married patients or patients with previous deliveries did have recurrent tumor.

b) Recurrence and tumor laterality: (Table 5)

$100 \%$ of recurrent tumor occurred in unilateral tumors, recurrent tumors were left sided in $100 \%$ of cases. None of the bilateral tumors did recur.

c) Recurrence and IOTA scoring system: (Table 6)

IOTA M1and M5 features was negative in all cases. M2 feature was present in $100 \%$ of cases who had recurrent tumors. M3 and M4 were positive in $66.7 \%$ and $33.3 \%$ of recurrent cases respectively.

Table 3. Tumor recurrence rates.

\begin{tabular}{ccc}
\hline Recurrence & Frequency & Percent \\
\hline No & 21 & 87.5 \\
Yes & 3 & 12.5 \\
\hline
\end{tabular}

Table 4. Type of surgical procedure used in FSS. SO: salpingo-oophorectomy.

\begin{tabular}{ccc}
\hline Surgical procedure & Frequency & Percent \\
\hline Unilateral SO & 20 & 84.3 \\
Unilateral SO, contralateral cystectomy & 4 & 16.6 \\
\hline
\end{tabular}


Table 5. Relation between recurrence rate, demographic data and tumor laterality.

\begin{tabular}{|c|c|c|c|c|c|}
\hline \multirow{3}{*}{ Crosstab } & \multicolumn{4}{|c|}{ Recurrence } & \multirow{3}{*}{$\begin{array}{c}\mathrm{X}^{2} \\
\mathrm{P}\end{array}$} \\
\hline & \multicolumn{2}{|c|}{ No } & \multicolumn{2}{|c|}{ Yes } & \\
\hline & No & $\%$ & No & $\%$ & \\
\hline Age & \multicolumn{2}{|c|}{$24.43 \pm 8.54$} & \multicolumn{2}{|c|}{$23.33 \pm 1.15$} & 0.830 \\
\hline Married & 13 & 61.9 & 0 & 0.0 & 4.052 \\
\hline Virgin & 8 & 38.1 & 3 & 100.0 & 0.082 \\
\hline \multicolumn{6}{|c|}{ Gravidity and Parity } \\
\hline 0 & 17 & 80.9 & 3 & 0.0 & \multirow{3}{*}{$\begin{array}{l}13.029 \\
0.011^{\star}\end{array}$} \\
\hline 1 & 2 & 9.5 & 0 & 0.0 & \\
\hline \multirow[t]{2}{*}{3} & 2 & 9.5 & 0 & 0.0 & \\
\hline & \multicolumn{4}{|c|}{ Laterality } & \\
\hline Bilateral & 4 & 19.0 & 0 & 0.0 & 0.686 \\
\hline Unilateral & 17 & 81.0 & 3 & 100.0 & 0.563 \\
\hline
\end{tabular}

Table 6. Relation between recurrence and IOTA score.

\begin{tabular}{|c|c|c|c|c|c|}
\hline & \multicolumn{4}{|c|}{ Recurrence } & \multirow{3}{*}{$\mathbf{P}$} \\
\hline & \multicolumn{2}{|c|}{ No } & \multicolumn{2}{|c|}{ Yes } & \\
\hline & No & $\%$ & No & $\%$ & \\
\hline IOTA M1 & 8 & 38.1 & 0 & 0.0 & 0.277 \\
\hline IOTA M2 & 13 & 61.9 & 3 & 100.0 & 0.277 \\
\hline IOTA M3 & 7 & 33.3 & 2 & 66.7 & 0.308 \\
\hline IOTA M4 & 8 & 38.1 & 1 & 33.3 & 0.692 \\
\hline IOTA M5 & 12 & 57.1 & 0 & 0.0 & 0.109 \\
\hline
\end{tabular}

Table 7. Recurrence and type of surgical procedure, tumor HP and tumor grade.

\begin{tabular}{|c|c|c|c|c|c|}
\hline \multirow{4}{*}{ surgical procedure } & \multicolumn{4}{|c|}{ Recurrence } & \multirow{4}{*}{$\begin{array}{c}X^{2} \\
P\end{array}$} \\
\hline & \multirow{2}{*}{\multicolumn{2}{|c|}{$\begin{array}{c}\text { No } \\
\text { “n }=21 "\end{array}$}} & \multirow{2}{*}{\multicolumn{2}{|c|}{$\begin{array}{c}\text { Yes } \\
\text { “n = 3" }\end{array}$}} & \\
\hline & & & & & \\
\hline & No & $\%$ & No & $\%$ & \\
\hline \multirow{3}{*}{$\begin{array}{c}\text { lt.SO } \\
\text { Lt.SO } \\
\text { Unilateral SO and contralateral } \\
\text { cystectomy }\end{array}$} & 11 & 52.4 & 0 & 0.0 & \multirow{4}{*}{$\begin{array}{l}24.000 \\
0.001^{*}\end{array}$} \\
\hline & 0 & 0.0 & 3 & 100.0 & \\
\hline & 4 & 19 & 0 & 0.0 & \\
\hline \multirow[t]{2}{*}{ Rt.SO } & 6 & 28.6 & 0 & 0.0 & \\
\hline & \multicolumn{4}{|c|}{ Tumor histopathological type } & \multirow{4}{*}{$\begin{array}{l}2.449 \\
0.294\end{array}$} \\
\hline Epithelial & 11 & 52.4 & 3 & 100.0 & \\
\hline Six cord stromal tumor & 6 & 28.6 & 0 & 0.0 & \\
\hline \multirow[t]{2}{*}{ Germ cell tumour } & 4 & 19.0 & 0 & 0.0 & \\
\hline & \multicolumn{3}{|c|}{ Tumor grade } & & \\
\hline Grade 1 & 6 & 28.6 & 0 & 0.0 & \multirow{4}{*}{$\begin{array}{l}7.543 \\
0.110\end{array}$} \\
\hline Grade 2 & 7 & 33.3 & 1 & 33.3 & \\
\hline Grade 3 & 2 & 9.5 & 2 & 66.7 & \\
\hline No & 6 & 28.6 & 0 & 0.0 & \\
\hline
\end{tabular}


d) Recurrence and type of surgical procedure: (Table 7)

Recurrence was reported in 3/24 of cases (12.5\%), such 3 cases underwent unilateral So plus a FCSLP. No recurrence was reported in cases bilateral tumors who underwent unilateral $\mathrm{SO}$ and a contralateral cystectomy.

e) Recurrence and tumor HP and tumor grade: (Table 7)

$100 \%$ of recurrence was in epithelial tumors. $1 / 24$ (4.1\%) was clear cell, $1 / 24$ (4.1\%) was serous and 1/24 (4.1\%) was mucinous. None of the endometroid tumors did recur. Also none of the non-epithelial tumors showed any recurrence. Tumors of G1 showed no recurrence, G2 tumors showed 33.3\% recurrence and G3 tumors showed the highest recurrence rate (66.6\%).

\section{Discussion}

Ovarian cancer is a disease of postmenopausal women. About $15 \%$ of OC cases occur in adolescence and in women in younger age groups [2]. The primary surgical treatment of OC includes a full staging laparotomy procedure (FSLPs) which includes TAH with BSO, infracolic omentectomy (which may proceed to supracolic or total omentectomy), random peritoneal biopsies and pelvic and paraaortic lymph node sampling. This will result in loss of reproductive as well as hormonal capabilities [10] [11].

Ovarian FSS are group of surgical procedures which can be done in selected patients with apparently early (stage 1) OC to keep their reproductive potential and hormonal function [11]. These FSS should be accompanied by a comprehensive surgical staging or a full conservative staging procedure (FCSLP). The value of FCSLP is to detect any microscopic or occult metastasis in omentum, lymph nodes or peritoneum which, if present, will halt the conservative treatment. Candidates of such FSS include low risk early OC patients including early epithelial OC (non-clear cell types which include low grade mucinous and endometroid types), diploid tumor variants, FIGO sub-stage $1(1 \mathrm{a}, 1 \mathrm{~b})$ and low levels of CA125 in post-operative follow-up [12]. Other types of non-epithelial OC can be treated by such methods like some of ovarian germ cell tumors and some sex cord stromal tumors [13].

All our cases had undergone a FCSLP with all steps mentioned above. In contrast to simple unilateral SO, the fertility-preserving staging operation is more aggressive, and postoperative pelvic adhesions may be more common, which might be considered a mechanical cause of infertility. A study by Yong-Soon Kwon (2009), et al. that there was no recurrence was reported after treatment with such FCSLP of low risk patients. [14]. We reported the same results that no recurrence was reported after treatment of the same patients with low risk factors.

Most of our patients were stage 1a in $83.3 \%$ of cases (Table 2). Same results were also reported by Fakhr et al. [15] which explains the low recurrence in those cases. None of our cases was found to have positive lymph node (LN) metastasis (Table 2) In contrast, Cass I, (2001) et al. and several authors found that 
$15 \%$ of his patients had positive LN involved by the tumor [16]. This is called (occult stage 3 ), hence the importance of the complete surgical staging procedure.

\section{Conclusion}

To summarize our results, we can say that ovarian FSS is a safe surgical option for nearly all OC patients with low risk of recurrence, with apparently early stage OC, after being confirmed by a FCSLP to exclude any occult metastasis (occult advanced stage OC), including those cases of early epithelial OC, germ cell and gonadal stromal tumors and it should be considered for patients who have a strong desire to keep their fertility.

\section{Conflicts of Interest}

The author declares no conflicts of interest regarding the publication of this paper.

\section{References}

[1] Bray, F., Ferlay, J., Laversanne, M., et al. (2015) Cancer Incidence in Five Continents: Inclusion Criteria, Highlights from Volume X and the Global Status of Cancer Registration. International Journal of Cancer, 137, 2060-2071. https://doi.org/10.1002/ijc.29670

[2] Abbott, D.W., Freeman, M.L. and Holt, J.T. (1998) Double-Strand Break Repair Deficiency and Radiation Sensitivity in BRCA2 Mutant Cancer Cells. Journal of the National Cancer Institute, 90, 978-985. https://doi.org/10.1093/jnci/90.13.978

[3] Jemal, A., Murray, T. and Thun, M. (2003) Cancer Statistics, 2003. CA: A Cancer Journal for Clinicians, 53, 5-26. https://doi.org/10.3322/canjclin.52.1.23

[4] Schneider, D.T., Calaminus, G., Wessalowski, R., Pathmanathan, R., Selle, B., Sternschulte, W., et al. (2003) Ovarian Sex Cord-Stromal Tumors in Children and Adolescents. Journal of Clinical Oncology, 21, 2357-2363. https://doi.org/10.1200/JCO.2003.05.038

[5] Young, R.H. and Scully, R.E. (1984) Well-Differentiated Ovarian Sertoli-Leydig Cell Tumors: A Clinico-Pathological Analysis of 23 Cases. International Journal of $G y$ necological Pathology, 3, 277-290. https://doi.org/10.1097/00004347-198403000-00004

[6] Liu, D.H., Cai, J., Gao, A.W., Wang, Z.H., et al. (2020) Fertility Sparing Surgery vs Radical Surgery for Epithelial Ovarian Cancer: A Meta-Analysis of Overall Survival and Disease-Free Survival. BMC Cancer, 20, 320.

[7] Martín-Cameán, M., Delgado-Sánchez, E., et al. (2016) The Role of Surgery in Advanced Epithelial Ovarian Cancer. Ecancermedicalscience, 10, 666. https://doi.org/10.3332/ecancer.2016.666

[8] Trimbos, J.B., Parmar, M., Vergote, I., et al. (2003) International Collaborative Ovarian Neoplasm Trial 1 and Adjuvant Chemotherapy in Ovarian Neoplasm Trial: Two Parallel Randomized Phase III Trials of Adjuvant Chemotherapy in Patients with Early-Stage Ovarian Carcinoma. Journal of the National Cancer Institute, 95, 105-112. https://doi.org/10.1093/inci/95.2.105

[9] Timmerman, D., Ameye, L., Fischerova, D., et al. (2010) Simple Ultrasound Rules to 
Distinguish between Benign and Malignant Adnexal Masses before Surgery: Prospective Validation by IOTA Group. BMJ, 341, c6839.

https://doi.org/10.1136/bmj.c6839

[10] Colombo, N., Chiari, S., Maggioni, A., Bocciolone, L., Torri, V. and Mangioni, C. (1994) Controversial Issues in the Management of Early Epithelial Ovarian Cancer: Conservative Surgery and Role of Adjuvant Therapy. Gynecologic Oncology, 55, S47S51. https://doi.org/10.1006/gyno.1994.1341

[11] Zanetta, G., Chiari, S., Rota, S., Bratina, G., Maneo, A., Torri, V., et al. (1997) Conservative Surgery for Stage I Ovarian Carcinoma in Women of Childbearing Age. British Journal of Obstetrics and Gynaecology, 104, 1030-1035. https://doi.org/10.1111/j.1471-0528.1997.tb12062.x

[12] Vergote, I., Brabanter, J., Fyles, A., Bertelsen, K., Einhorn, N., Sevelda, P., et al. (2000) Prognostic Importance of Degree of Differentiation and Cyst Rupture in Stage I Invasive Epithelial Ovarian Carcinoma. The Lancet, 357, 176-182. https://doi.org/10.1016/S0140-6736(00)03590-X

[13] Kurman, R.J. and Norris, H.J. (1977) Malignant Germ Cell Tumors of the Ovary. Human Pathology, 8, 551-564. https://doi.org/10.1016/S0046-8177(77)80115-9

[14] Kwon, Y.-S., Hahn, H.-S., Kim, T.-J., Lee, I.-H., Lim, K.-T., et al. (2009) Fertility Preservation in Patients with Early Epithelial Ovarian Cancer. Gynecologic Oncology, 20, 44-47. https://doi.org/10.3802/jgo.2009.20.1.44

[15] Fakhr, I., Abd-Allah, M., Ramzy, S., et al. (2013) Outcome of Fertility Preserving Surgery in Early Stage Ovarian Cancer. Journal of the Egyptian National Cancer Institute, 25, 219-222. https://doi.org/10.1016/j.jnci.2013.07.003

[16] Cass, I., Li, A.J., Runowicz, C.D., Fields, A.L., Goldberg, G.L., Leuchter, R.S., et al. (2001) Pattern of Lymph Node Metastases in Clinically Unilateral Stage I Invasive Epithelial Ovarian Carcinomas. Gynecologic Oncology, 80, 56-61. https://doi.org/10.1006/gyno.2000.6027 